\title{
Hybrid-view Programming of Nuclear Fusion Simulation Code in the PGAS Parallel Programming Language XcalableMP
}

\author{
Keisuke Tsugane ${ }^{\mathrm{a}, *}$, Taisuke Boku ${ }^{\mathrm{a}, \mathrm{b}}$, Hitoshi Murai ${ }^{\mathrm{c}}$, Mitsuhisa Sato ${ }^{\mathrm{a}, \mathrm{c}}$, \\ William Tang ${ }^{\mathrm{d}, \mathrm{e}}$, Bei Wang ${ }^{\mathrm{e}}$ \\ ${ }^{a}$ Graduate School of Systems and Information Engineering, University of Tsukuba, 1-1-1 \\ Tennodai, Tsukuba, Ibaraki, 305-8573, Japan \\ ${ }^{b}$ Center for Computational Sciences, University of Tsukuba, 1-1-1 Tennodai, Tsukuba, \\ Ibaraki, 305-8577, Japan \\ ${ }^{c}$ Advanced Institute for Computational Science, RIKEN, 7-1-26, \\ Minatojima-minami-machi, Chuo-ku, Kobe, Hyogo, 650-0047, Japan \\ ${ }^{d}$ Princeton Plasma Physics Laboratory, Princeton University, 100 Stellarator Rd Princeton, \\ NJ 08540, USA \\ ${ }^{e}$ Princeton Institute for Computational Science and Engineering, Princeton University, 335 \\ Lewis Science Library Washington Road and Ivy Lane Princeton, NJ 08544, USA
}

\begin{abstract}
Recently, the Partitioned Global Address Space (PGAS) parallel programming model has emerged as a usable distributed memory programming model. XcalableMP (XMP) is a PGAS parallel programming language that extends base languages such as $\mathrm{C}$ and Fortran with directives in OpenMP-like style. XMP supports a global-view model that allows programmers to define global data and to map them to a set of processors, which execute the distributed global data as a single thread. In XMP, the concept of a coarray is also employed for local-view programming. In this study, we port Gyrokinetic Toroidal Code - Princeton (GTC-P), which is a three-dimensional gyrokinetic PIC code developed at Princeton University to study the microturbulence phenomenon in magnetically confined fusion plasmas, to XMP as an example of hybrid memory model coding with the global-view and local-view programming models. In local-view programming, the coarray notation is simple and intuitive compared with Message Passing Interface (MPI) programming while the performance is comparable to that of the MPI version. Thus, because the global-view programming model is suitable for expressing the data parallelism for a field of grid space data, we implement a hybrid-view version using a global-view programming model to compute the field and a local-view programming model to compute the movement of particles. The performance is degraded by $20 \%$ compared with the original MPI version, but the hybrid-view version facilitates more natural data expression for static grid space data (in the global-view model) and
\end{abstract}

* Corresponding author. Tel.:+81.29.853.6912; fax.:+81.29.853.6912.
Email address: tsugane@hpcs.cs.tsukuba.ac.jp (Keisuke Tsugane)

Preprint submitted to Journal of ${ }^{A} T_{E} X$ Templates

May 26, 2016

(C) 2016. This manuscript version is made available under the Elsevier user license http://www.elsevier.com/open-access/userlicense/1.0/ 
dynamic particle data (in the local-view model), and it also increases the readability of the code for higher productivity.

Keywords: GTC-P, Partitioned Global Address Space, Gyrokinetic PIC code, Nuclear Fusion Simulation, XcalableMP

2010 MSC: 00-01, 99-00

\section{Introduction}

The Message Passing Interface (MPI) is used widely as a parallel programming model for distributed memory systems. However, its low productivity due to complex local array indexing and error-prone coding during communication

5 is a huge problem because it forces programmers to describe the data distribution explicitly and they must employ inter-node communication using primitive API functions.

Recently, the Partitioned Global Address Space (PGAS) parallel programming model has emerged as a usable distributed memory programming model.

10 A PGAS language model contains a global address space where any processes can view the data globally in a similar manner to a shared memory system. The global address space is logically partitioned into each process, which can access its own data more efficiently, thereby allowing programmers to perform locality-aware parallel programming.

15 PGAS parallel programming languages such as UPC[1], Coarray Fortran[2], and X10[3] have been proposed based on this model. XcalableMP[4][5][6], or XMP for short, is another PGAS parallel programming language, which is an extension of existing languages such as $\mathrm{C}$ and Fortran with directives similar to OpenMP. XMP enables the parallelization of serial source code with additional 20 directives to describe the data distribution, synchronization, and consistency among them over multiple nodes with distributed memory architecture. This approach to extending base languages ( $\mathrm{C}$ or Fortran) with directives makes it easier for programmers to develop parallel programs on distributed memory systems.

25 Some PGAS languages support the global-view model that allows programmers to define global data and to map them to a set of processors, which execute the distributed global data as a single thread. In XMP, the global-view model allows programmers to define global arrays, which are distributed to processors by adding the directives. Some typical communication patterns are supported

30 by directives, such as data exchange between neighbor processors in stencil computations.

In contrast to the global-view model, the local-view model describes remote memory access using the node (processor) index. This operation is implemented as one-sided communication. XMP employs the coarray concept from Coarray

35 Fortran as a local-view programming model. A coarray is a distributed data object, which is indexed by the coarray dimension that maps indices to processors. In XMP, the coarray is defined in $\mathrm{C}$ as well as Fortran. In the local-view 
model, a thread on each processor executes its own local computations independently with remote memory access to data located in different processors by 40 coarray access. The local-view model requires that programmers define their algorithms by explicitly decomposing the data structures and controlling the flow in each processor. The data view is similar to that in MPI, but coarray remote access provides a more intuitive view of accessing the data in different processors, thereby increasing productivity.

45 In this study, we consider a hybrid-view programming approach, which combines the global-view and local-view models in XMP according to the characteristics of the distributed data structure of the target application. The globalview model allows programmers to express regular parallel computations such as domain decomposition with stencil computation in a highly intuitive manner so simply by adding directives to a serial version of code. However, it is difficult to describe parallel programs in the global-view model when more irregular communication patterns and complex load balancing are required on the processing. Thus, local-view programming is necessary in these situations.

We utilize this hybrid-view programming for Gyrokinetic Toroidal Code 55 Princeton (GTC-P)[7], which is a large-scale plasma turbulence code that can be applied at the International Thermonuclear Experimental Reactor (ITER [8]) scale and beyond for next-generation nuclear fusion simulation. The GTC$\mathrm{P}$ is an improved version of the original GTC[9] and it is a type of gyrokinetic Particle-in-Cell (PIC) code with two basic data arrays: global grid data that

60 corresponds to the physical problem space and particle data that corresponds to particles moving around the grid space. The original GTC-P was written in $\mathrm{C}$ as a form of hybrid programming with OpenMP and MPI. In this code, the grid data and particle data are mapped onto MPI processes and exchanged. As found with most codes of this type, it is difficult to manage complex data 65 distributions and communication for both grid data and particle data during code development. Furthermore, to simulate the microturbulence phenomenon in plasmas for magnetically confined fusion devices, non-flat domain decomposition is necessary in one dimension, as well as parallelizing multiple dimensions, to obtain accurate large-scale simulations. Therefore, the number of computa-

70 tions becomes extremely large for next-generation and large-scale reactors such as ITER.

We consider both types of data models in XMP, i.e., global-view and localview models, which are suitable for representing grid space data and particle data, respectively, because of their data distribution and communication pattern. In this study, we implement the GTC-P code in two ways: using XMP with a local-view only model, and with a combination of local-view and globalview models, where we evaluate the performance and productivity of these approaches. As the preliminary result, we implemented and evaluated the GTC-P in XMP hybrid-view model[10]. Moreover, we indicate the causes of perfor-

so mance degradation for GTC-P in XMP and evaluate the GTC-P of hybrid versions written in XMP+OpenMP and MPI+OpenMP in this study.

The remainder of this paper is organized as follows. We provide the related research and an overview of XMP with an example of code in Section 2 and 
Section 3, and we briefly describe the GTC-P nuclear fusion simulation code in Section 4. Section 5 describes the implementation of GTC-P using XMP. We report the performance and productivity evaluation in Section 6 , and we conclude our study in Section 7.

\section{Related Research}

Chapel[11] is widely known as PGAS language having the global-view programming model designed and developed by Cray Inc. As a feature, Chapel employs the original language where programmers can describe several different parallelisms, such as inter/inner node, instruction level threads, and accelerator. In addition, the communication of data based on the global-view model is executed implicitly. While the automatic communication is useful for pro-

95 grammers without experience in parallel programming, it is difficult to tune its performance. In contrast, XMP is an extension of existing languages by directives. Therefore, it makes easier for programmers to extend existing serial code to parallel code. The communication is generated by directives to facilitate the performance tuning.

GTC or GTC-P have been executed and optimized on some platforms. X. Liao et al.[12] optimized GTC to use offload-programming model for the Intel Xeon Phi accelerator, and evaluated the performance on MilkyWay-2 supercomputer. K. Madduri et al.[13] described the optimization for multi- and manycore systems, and evaluated on some systems including Graphic Processing Unit

105 (GPU) based on NVIDIA Fermi architectures. In our study, we focus on the evaluation of not only the performance but also the productivity for GTC-P.

PIC method is often implemented some PGAS parallel programming languages. H. Sakagami and T. Mizuno[14] implemented 2D particle code, ESPAC2: 2D electro-static plasma, based on PIC method using High Performance ${ }_{110}$ Fortran (HPF) [15] which is directive-based language similar to OpenMP and supports the global-view model. The particle data is distributed into the block, while the electrostatic field is replicated onto each process. After the distributed particle data is calculated in each process, the reduction operation is executed to update the particle data of electrostatic field on each time step. The data distribution is an easy expression which is annotated by directives in HPF. R. Preissl et al.[16] introduced hybrid PGAS+OpenMP approach for 3D PIC code, Gyrokinetic Tokamak Simulation (GTS) which is implemented in MPI+OpenMP. As PGAS parallel programming language, they used Coarray Fortran. The onesided communication in Coarray Fortran is simple and more intuitive notation 120 compared with MPI programming because it is expressed in the form of array assignment statement. However, the description of data distribution is same as MPI. To use simple coarray communication and easy data distribution by directives, we consider a hybrid-view approach, which combines the global-view and local-view models in XMP. 


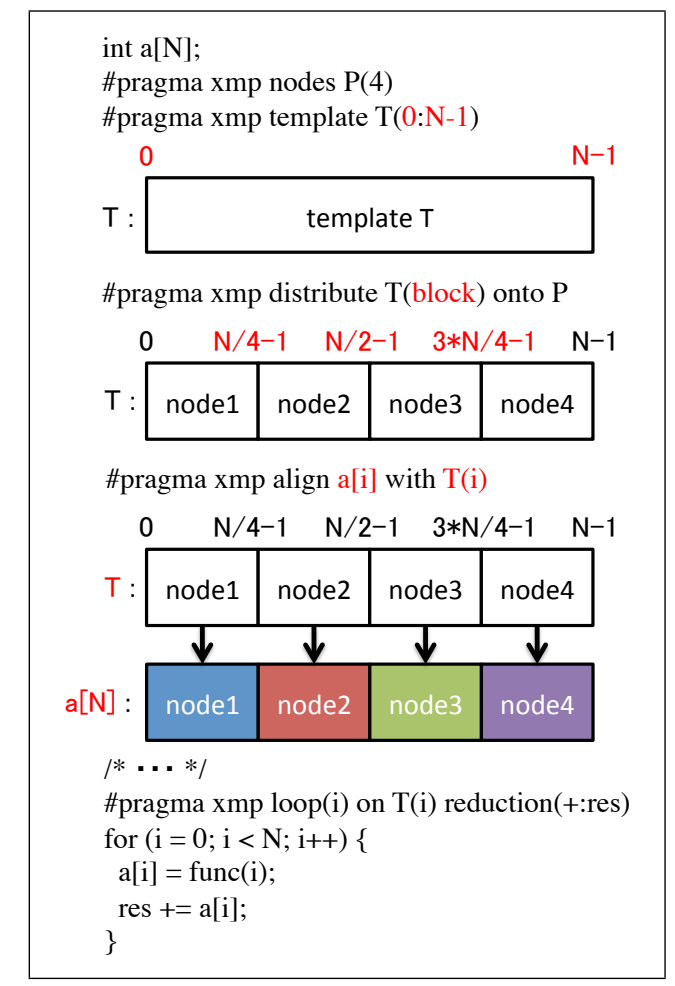

Figure 1: Example of a global-view programming model. these distributed data. This model makes it easy for users to program typical data parallel processing tasks based on a static data distribution. The data distribution uses a virtual array called a template. Figure 1 shows an example of 140

\section{Overview of XMP}

XMP was designed by the XMP Specification Working Group and its reference implementation, the Omni XcalableMP compiler, is being developed by the University of Tsukuba and the Advanced Institute for Computational Science, RIKEN[17]. The basic execution model is a Single Program Multiple Data (SPMD) that operates on a distributed memory architecture such as MPI. XMP supports OpenMP-like directives for data distribution, work mapping, communication, and synchronization. XMP provides global-view and local-view programming models, which can be used independently or combined.

\subsection{Global-view Programming Model}

The global-view programming model supports data distribution on computational nodes set at either run-time or compile-time, and parallel operations on a global-view programming model using the data distribution fixed at compiletime. First, the number of execution nodes (processes) and the template length 


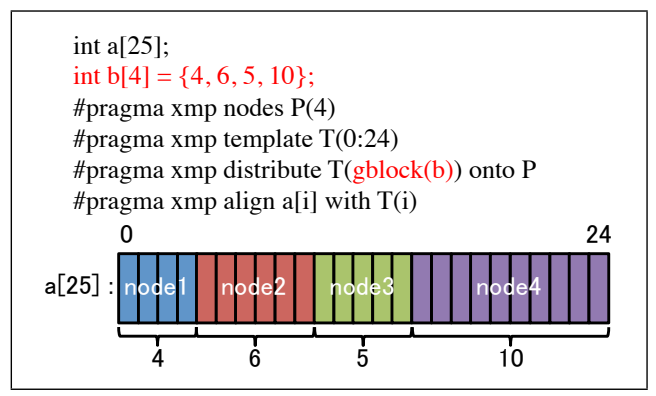

Figure 2: Example of a gblock distribution.

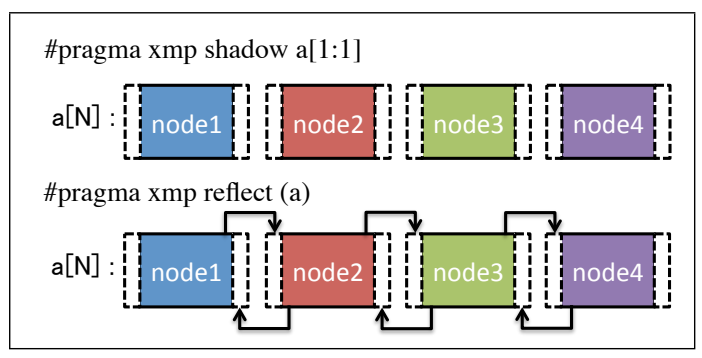

Figure 3: Examples of the shadow and reflect directives.

are determined by the node and template directives. Second, the distribution of the template is described by the distributed directive, which has a specific distribution format, such as a block, cyclic, block-cyclic, or gblock (as shown below). Third, an array is aligned with a template using the align directive. As a result, each element of the array is assigned via the distributed template. In this example, the loop directive is then executed in parallel by the executing nodes and collective communication is enabled by the reduction directive or the clause of the loop directive, as shown in Figure 1. Moreover, when these 150 directives can be ignored as a comment by the compilers, the XMP program is executed sequentially.

As a feature of the data distribution on XMP, there is a gblock notation which provides a user-defined block distribution at either run-time or compiletime mapping on computational nodes. Figure 2 shows the example of a gblock distribution fixed at compile-time. Each block sizes are stored in array b, and it is specified to distribute directive as an argument of a global notation.

Figure 3 shows an example of the shadow and reflect directives for data exchange between neighboring processors such as stencil computations. The shadow directive can be assigned to the sleeve area, as shown by the dotted line 160 elements of Figure 3, when the array is distributed to the block or gblock. In addition, the reflect directive[18] can be communicated to update the elements, which is assigned by the shadow directive. 


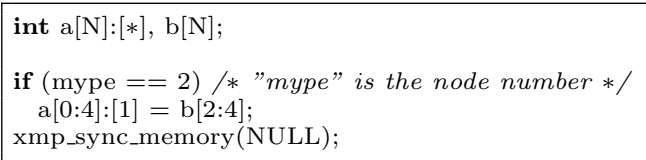

Figure 4: Example of a local-view programming model.

\subsection{Local-view Programming Model}

The local-view programming model supports a function that performs com-

165 munication for each node's local data. XMP employs the coarray notation as an extension of Coarray Fortran and the function is described in the form of an array assignment statement. Figure 4 shows a local-view programming model where the elements from 2 to 5 in the local array b of node 2 are communicated to local array a from 0 to 3 in node 1 . Therefore, the local-view programming is described, such as MPI point-to-point communication. Moreover, local-view programming model also provides some functions in order to allow asynchronous communication in the coarray notation. The xmp_sync_memory can be used to guarantee the completion of communication by the point-to-point processes, the

175 xmp_sync_all can be used to guarantee the completion of communication by all the related processes, and the xmp_sync_image can be used to guarantee the communication between the process specified as its argument and myself.

\subsection{Hybrid-view Programming Model}

XMP allows the use of hybrid-view programming, which combines the global180 and local-view models. The global-view model allows program press regular parallel computations, such as domain decomposition with stencil computation, in a highly intuitive manner simply by adding directives to a serial version of the code. On the other hand, when the data distribution cannot be simply described in domain decomposition manner or the communication pattern is complicated, the global-view model is not suitable, and more dynamism is required to express the code naturally. Thus, the coarray notation provided by the local-view model is required in this case, and it is possible to program in a flexible manner using these models.

\section{Nuclear Fusion Simulation Code}

Typical methods used to simulate the microturbulence phenomenon in magnetically confined fusion plasmas include the Monte Carlo method and the PIC method. In this study, we only consider the gyrokinetic PIC method among them as a target application to explain the GTC and GTC-P code briefly. 


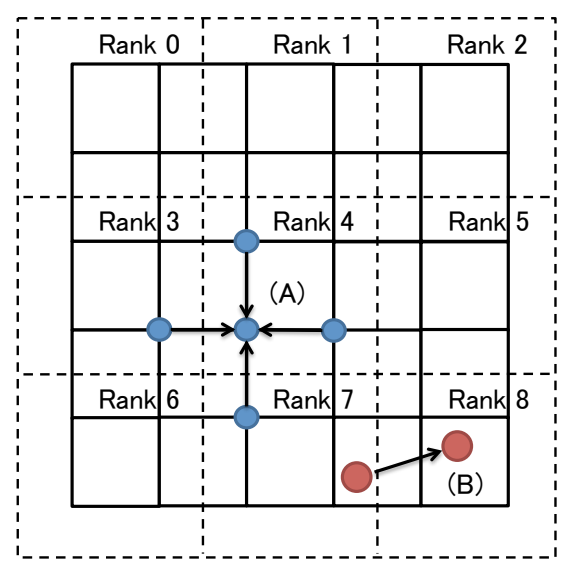

Figure 5: Image from a gyrokinetic PIC simulation with a two-dimensional block distribution; (A) Calculation of the field using the nearby grid points, and (B) the movement of a particle. The dashed lines indicate processor boundaries.

\subsection{Gyrokinetic PIC Simulation}

The simulation of the gyrokinetic PIC method uses a space grid to calculate the field and for the particle trajectory calculation, which does not depend on the grid when moving in the free space. Figure 5 shows an image of a gyrokinetic PIC simulation with a two-dimensional block distribution. The typical behavior of the gyrokinetic PIC code is as follows.

1. Add the charge of the particle to the nearby grid points.

2. Solve the electric field affected by the electrostatic potential by calculating the charge density of the nearby grid points using Poisson's equation.

3. Interpolate the electric field in the current position based on each particle in the nearby grid points and move the position of the particle in the space.

During each step, a process must communicate with another if that process holds the data for the space in the grid that is affected, as shown in Figure 5 (A), or if the particle data move from or to that process, as shown in Figure 5 (B). Based on the above, if the size of the distributed domain, e.g., the grid distribution employed in the global-view programming model is suitable and the communication between nearby grid points can be described by the reflect directive in XMP coding as described in Section 3.1. In contrast, if the number of particles on each distributed domain changes dynamically during each time step of the simulation, such as particle motion, coarray communication is required using local-view programming as described in Section 3.2. 


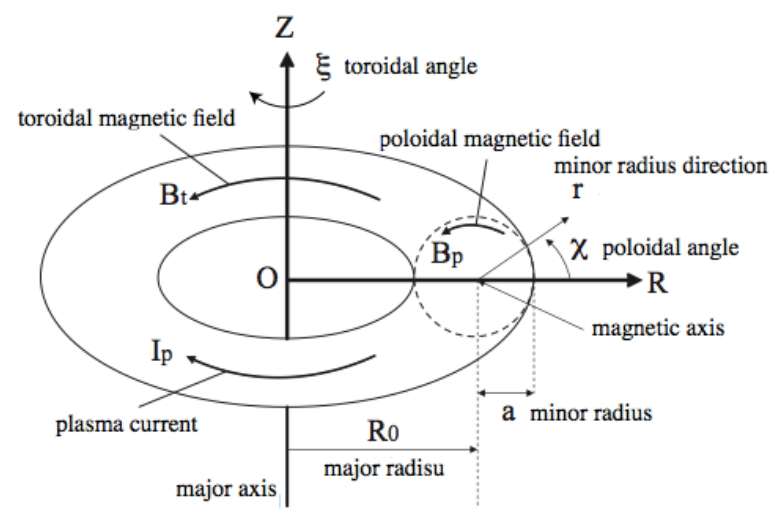

Figure 6: Conceptual image of the three-dimensional torus space in GTC-P[19].
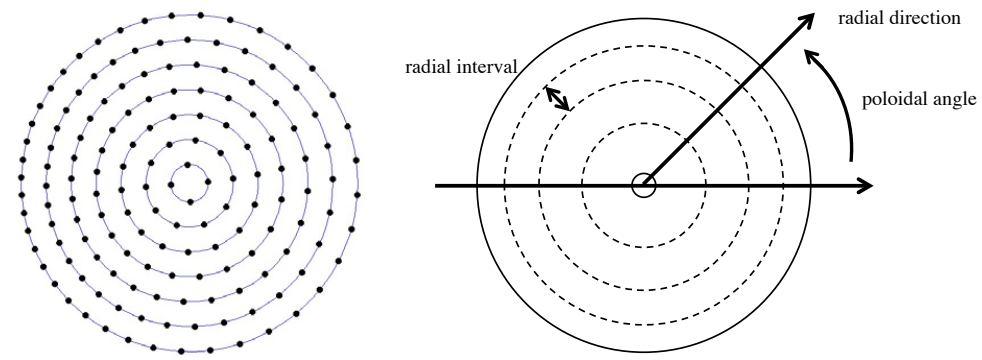

Figure 7: Example showing the grid points on the poloidal plane in GTC-P[20] (left). Image of the radial domain decomposition on poloidal plain. The dashed line shows the border of the decomposition (right).

\section{2. $G T C$}

GTC is a three-dimensional (3D) gyrokinetic PIC code, which was developed by DOE SciDAC, UC Irvine, etc.[9] for studying the microturbulence phenomenon in plasmas for magnetically confined fusion devices. Figure 6 shows a conceptual image of a 3D torus physical space. GTC treats the physical space and the movement of particles in three directions: the toroidal direction around the major axis, the poloidal direction around the magnetic axis, and the radial direction of the minor radius from the magnetic axis. The cross-section of the toroidal direction is known as the poloidal plane. GTC-P is a modified version of GTC, where there are several differences in the parallelization scheme. Moreover, GTC-P is implemented as two versions in the $\mathrm{C}$ and Fortran languages, whereas the original GTC is coded in Fortran. In this study, we focus on the C implementation of GTC-P.

230 GTC parallelizes the problem according to three levels. Processing on the space grid domain in the toroidal direction and the processing of particles in each 


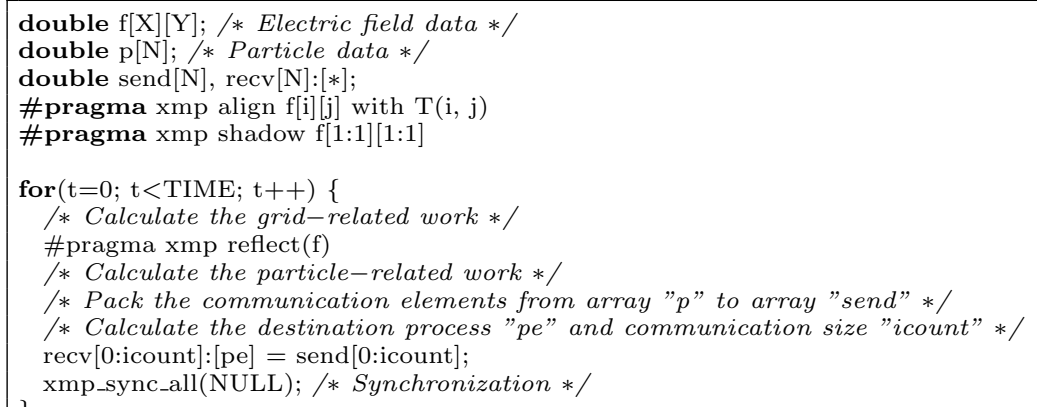

Figure 8: Example showing the implementation of a gyrokinetic PIC simulation with XMP.

domain are mapped onto MPI processes. Also, the grid-related calculation and particles in each distributed domain are further subdivided using OpenMP for each process. GTC-P has four levels of parallelism with additional parallelism in

235 the radial direction. The total number of MPI processes that need to be executed is $N_{t} \times N_{r} \times N_{r p}$, where $N_{t}$ is the number of domains decomposed in the toroidal direction, $N_{r}$ is the number of domains decomposed in the radial direction, and $N_{r p}$ is the number of particles decomposed in each of the distributed domains.

There is a difference in the number of grid points on the poloidal plane, as demonstrated in Figure 7 (left). The toroidal domain can be distributed with equally sized intervals, but the radial domain cannot be distributed with equally sized intervals due to the large difference in the domain size depending on its position in space. Therefore, in order to align as much as possible the number of grid points to be mapped on each process, the outer area of the radial domain 245 is distributed as short radial interval and its inner area is distributed as long radial interval, such as Figure 7 (right).

GTC-P has mainly six computational kernels. The charge kernel deposits the charge from particles onto the grid using the 4-point approximation of nearby grid points. The poisson, field, and smooth kernels solve the gyrokinetic Pois250 son's equation, compute an electric field, and smooths the charge and potential with a filter on the grid, respectively. The push kernel interpolates the electric field onto particles using the field. The charge and push kernels account for large percentage of the elapsed time in this simulation [7][21].

\section{Implementation of GTC-P by Hybrid-view Programming}

255 In this section, we indicate how to implement GTC-P using XMP. Figure 8 shows a skeleton code of the implementation of a gyrokinetic PIC simulation with XMP. In this example, the grid uses a two-dimensional block distribution and each block has a sleeve area, which is used to calculate the field with the nearby grid points based on the shadow directive. The particle movement

is represented by the coarray notation where the communication elements are 


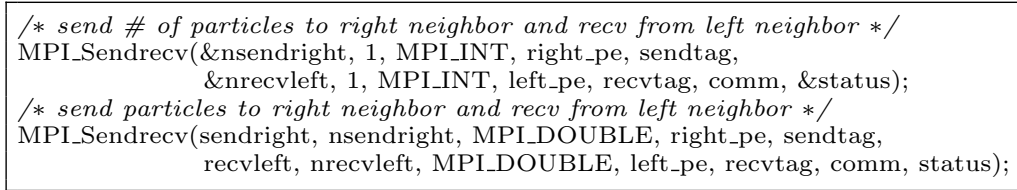

Figure 9: Particle data movement using MPI point-to-point communication in GTC-P

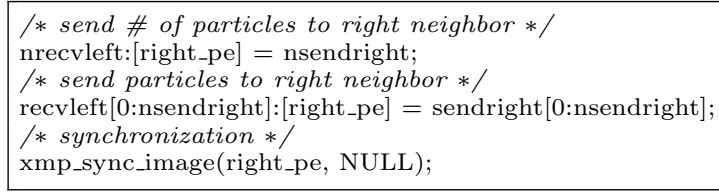

Figure 10: Particle data movement using the coarray notation in GTC-P

packed in the array send. Based on the above, we describe the two implementations of GTC-P using XMP. First, we implement the XMP-localview version using coarray communication, which is equivalent to using MPI point-to-point communication with the exception of MPI collective communication (as shown below). Next, the XMP-hybridview version is implemented by describing the fields using a distributed array with the reflect directive for overlapped sleeve area communication and the distributed data in the global-view programming model, as well as using the coarray notation to move the particle data. In addition, we use the bcast and reduction directives instead of MPI collective communication (MPI_Bcast and MPI_Allreduce) in both versions.

\subsection{Implementation Based on the XMP Local-view Model: XMP-localview}

In GTC-P, the communication processes required to move particles between grids and to exchange grid points are represented by MPI_Sendrecv or MPI_Isend/Irecv, where most of the communication is performed between ad-

275 jacent processes in one dimension. GTC-P has the steady state exchange of particles between neighboring subdomains. Because the number of particles changes dynamically, this implementation uses the coarray notation in the localview programming model.

Figures 9 and 10 show the particle data movement using MPI and the corresponding the coarray notation in GTC-P, respectively. In the exchange of coarray notation for particle data movement, it communicates the number of particles and the particle data, i.e., nsendright and sendright, with the adjacent process on the neighbor to the right. In addition, Figures 11 and 12 show the exchange of grid points using MPI and the corresponding coarray notation 285 in GTC-P, respectively. In the example of the coarray notation for the exchange of grid points, after copying a value to a one-dimensional array, i.e., sendr or $\mathrm{Xsendr}$, it communicates with the adjacent process on the neighbor to the right. Because the coarray notation is non-blocking communication, xmp_sync_image 


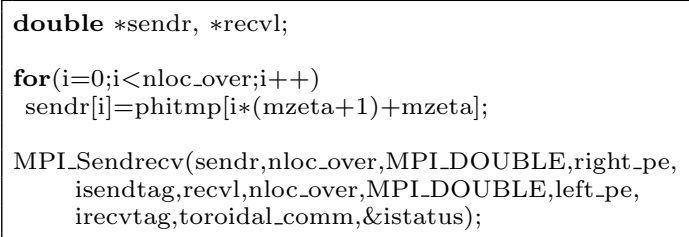

Figure 11: Exchange of grid points using MPI point-to-point communication in GTC-P.

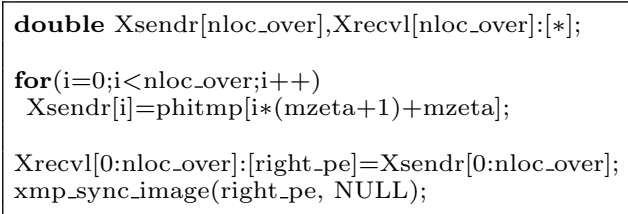

Figure 12: Exchange of grid points using the coarray notation in GTC-P.

1 \#pragma xmp reflect (phitmp) width $(0, /$ periodic/1:0)

Figure 13: Exchange of grid points using the reflect directive in GTC-P.

on the sixth line of Figure 10 and the seventh line of Figure 12 are required to 290 guarantee that communication has been completed between two processes, in this case, the neighboring (right_pe) and current processes.

\subsection{Implementation Based on the XMP Hybrid-view Model: XMP-hybridview}

In the XMP-hybridview implementation, all of the space grid data are denoted by a global-view model with compile-time mapping and the sleeve data are exchanged by XMP directives, whereas the particle data movements are denoted by a local-view model with the coarray notation, as shown Figure 10. As described in Section 4.2, it is necessary to represent an unequal block size for domain decomposition in the radial dimension. Because this dimension's space grid is denoted in the global-view model, we apply the gblock notation to represent it correctly in the same manner as the original MPI implementation. As shown in Section 3.1, the gblock notation can control the variable block size of each domain on the mapped space position. This feature is especially important for porting GTC-P onto XMP with a global-view model. Figure 14 shows an example of the GTC-P implementation with the XMP global-view program305 ming model using gblock. The 11th line of this example denotes the block size distribution in the radial dimension. Because of describing the data distribution by global-view model, we can describe the loop distribution only to insert loop directive onto the serial code that is from the 28th to 30th lines of this example. In addition, OpenMP directives can be combined with XMP such as the 27th line. 


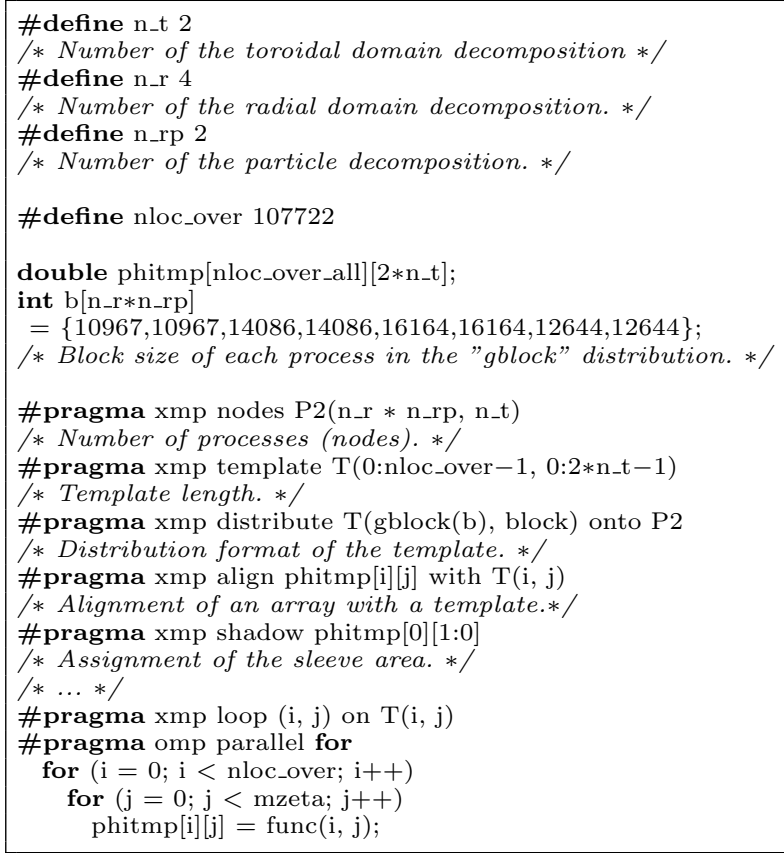

Figure 14: Example showing GTC-P implementation using the XMP global-view programming model.

The calculation of the grid-related works, such as the deposit of the charge from particles onto the grid using a 4-point approximation of grid points, the computation an electronic field, and the interpolation of the electronic field onto particles, are similar to 4-point stencil calculation on the poloidal plain. In

315 these codes, we can describe the loop parallelization by inserting loop directive onto the serial version. Appropriate directives are used for each dimension of the distributed array in XMP, and we further synchronize the sleeve data that overlap at each end of the distributed domain, which we can describe simply using the reflect directive. Figure 13 shows an example of the reflect directive, which is the same as the communication described in Figures 11 and 12. Thus, we can describe it using a directive on one line, which is much simpler compared with the MPI notation in Figures 11 and 12. When the width clause is specified, it can be designated as part of the sleeve elements and the periodic is used to update the sleeve area of the global lower (upper) bound based on the global upper (lower) bound.

\section{Performance Evaluation}

\subsection{Experimental Setting}

We evaluated the performance of our two implementations using a massively parallel GPU cluster: HA-PACS[22] at the Center for Computational Sciences, 


\begin{tabular}{l|l} 
Table 1: Machine Environment (HA-PACS Cluster) \\
\hline \hline CPU & Intel Xeon E5-2670 x2 (2.6GHz) \\
& CPU (8 cores/CPU) x2 = 16 cores \\
Memory & $128 \mathrm{~GB}$, DDR3 $1600 \mathrm{MHz}$ \\
Interconnection & Infiniband : Mellanox Connect-X3 \\
& Dual-port QDR \\
OS & CentOS 6.4 \\
C Compiler & gcc 4.4.7 \\
MPI & MVAPICH2 2.0 \\
GASNet & 1.24 .0 \\
\hline
\end{tabular}

Table 2: The amount of the memory usage for several different programming models (KB).

\begin{tabular}{c|c|c}
\hline \hline MPI & local-view & global-view \\
\hline 19488 & 19532 & 19888 \\
\hline
\end{tabular}

University of Tsukuba. Table 1 shows the computing environment employed for one node. HA-PACS is a GPU cluster, but we only utilized CPUs in this study, although we aim to extend our implementation with GPU acceleration in the near future, which is why we decided to use this machine as our platform. We apply the optimization option for NUMA with 'numactl --localalloc', and disable the CPU affinity setting of MVAPICH2 with MV2_ENABLE_AFFINITY=0.

As preliminary evaluations, we investigate the amount of the memory usage and the performance of communication using XMP and MPI. First, we indicate the comparison of the memory usage when one array is allocated in the localview model, global-view model, and MPI. They are evaluated with 'getpid()' and 'grep VmHWM /proc/[pid]/status' from C program during execution. An array size is $1 \mathrm{MB}$. We show the minimum size in the each amount of the memory usage when 4 node execution. The tests showed that the amount of memory usage of all programming models is almost same according to Table 2. Then, we evaluate the performance of XMP and MPI communication with Ping345 Pong program, which is defined by a power of two communication size, because XMP coarray is implemented by GASNet[23] which is a communication library optimized for some interconnections, e.g., Infiniband and Gemini. Figure 15 shows the performance of XMP coarray and MPI_Send/Recv communication. $\mathrm{XMP}$ is a good performance if the transfer size is about 65536 Bytes or less, whereas MPI is a good performance if it is more than about 65536 Bytes. We used a parameter of GASNet GASNET_IBV_PORTS="mlx4_0 $: 1+\mathrm{ml} \times 4 \_0: 2$ " which shows to use two ports of Infiniband, but we could get the performance of only single port of Infiniband. It may be an issue with GASNet library.

The GTC-P simulation size is determined by several important numerical 355 parameters. Table 3 shows the default parameters for problem size A provided by GTC-P, where we modified the parameters to evaluate weak scaling based on problem size A. Strong scaling was evaluated using the minimum parameters in the decomposition of each domain shown in Table 3, where mstep is the number of calculation steps, mzetamax is the number of grid points in the toroidal 


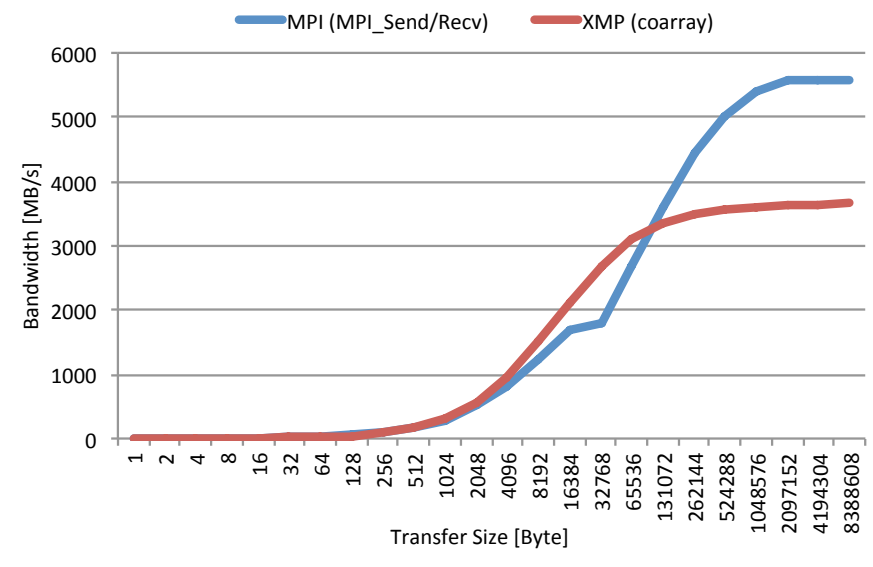

Figure 15: Ping-Pong communication bandwidth with MPI (MPI_Send/Recv) and XMP (coarray).

Table 3: Evaluation of the weak scaling of decomposition for each domain using problems ranging from 16 to 512 processes.

\begin{tabular}{l|c|c|c|c}
\hline \hline Problem Size A & Default & Toroidal & Radial & Particle \\
\hline mstep & 100 & 20 & 20 & 20 \\
mpsi & 90 & 90 & $90-2880$ & 90 \\
mzetamax & 64 & $2-64$ & 2 & 2 \\
particles per cell & 100 & 100 & 100 & $100-3200$ \\
\hline
\end{tabular}

360 the number of grid points in the poloidal plane and in the toroidal domain must be the same during decomposition, this was also changed in the parameter set for problem size A.

First, we used up to 32 nodes of HA-PACS where 16 processes ran on each mapped to evaluate the decomposition on each domain are shown in Table 4. As described above, three problem dimensions were considered: toroidal, radial, and particle. When we decomposed these dimensions into parallel processes, we always fixed the decomposition number on two dimensions (e.g., toroidal and 370 radial) as $2 \times 2$ and we varied the decomposition size in the other dimension (e.g., particle) from 4 to 128 , thereby scaling the total number of processes from 16 to 512. However, during decomposition on the toroidal dimension, we fixed the decomposition number on the radial and particle dimensions as $2 \times$ 4. This was due to variations in the number of calculations because increasing 375 the toroidal dimension also changes the poloidal planes, as described above. We used this scheme to change the scaling dimension.

Second, we used 16 nodes where one process ran on each node and the number of threads ranged from 1 to 16 in each process. The processes mapped 
Table 4: Process mapping to evaluate the scaling of decomposition for each domain $\left(N_{t} \times N_{r} \times N_{r p}\right)$.

\begin{tabular}{l|c|c|c}
\hline \hline Processes & Toroidal & Radial & Particle \\
\hline 16 & $2 \times 2 \times 4$ & $2 \times 4 \times 2$ & $2 \times 2 \times 4$ \\
32 & $4 \times 2 \times 4$ & $2 \times 8 \times 2$ & $2 \times 2 \times 8$ \\
64 & $8 \times 2 \times 4$ & $2 \times 16 \times 2$ & $2 \times 2 \times 16$ \\
128 & $16 \times 2 \times 4$ & $2 \times 32 \times 2$ & $2 \times 2 \times 32$ \\
256 & $32 \times 2 \times 4$ & $2 \times 64 \times 2$ & $2 \times 2 \times 64$ \\
512 & $64 \times 2 \times 4$ & $2 \times 128 \times 2$ & $2 \times 2 \times 128$ \\
\hline
\end{tabular}

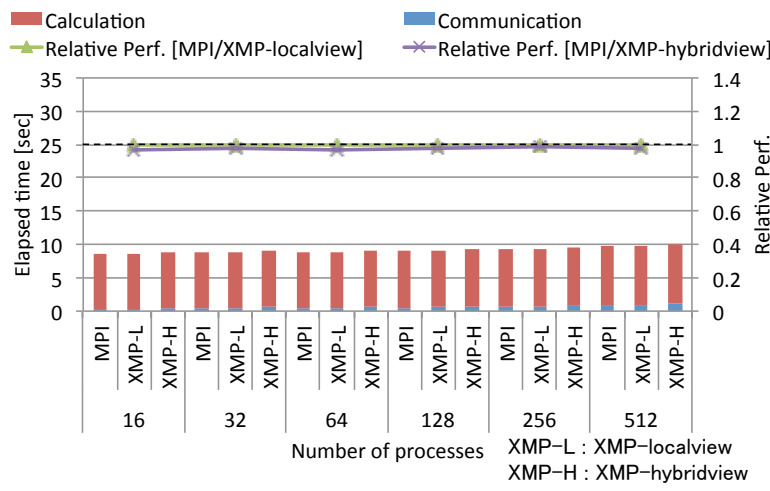

Figure 16: Elapsed time of the decomposition on toroidal dimension from 16 to 512 processes in weak scaling.

on each domain to evaluate the decomposition are $2 \times 4 \times 2$ and $2 \times 2 \times 4$.

\subsection{Results}

With weak scaling, Figures 16, 17 and 18 shows the elapsed time for both calculation and communication of MPI, XMP-localview and XMP-hybridview required to scale the number of processes from 16 to 512, where decomposition on the toroidal and particle dimensions exhibited good scalability whereas scaling on the radial dimension was poor. Figure 17 shows that the performance of decomposition on the radial dimension decreased as the number of nodes increased compared with the other two types of domain decomposition, as shown in Figures 16 and 18. Most of the communications are performed at the neighboring surface during decomposition on any dimension and the total amount of communication data does not vary greatly; thus, we focused on the calculation load balance between processes. Table 5 shows the difference between the maximum and minimum calculation times required for each type of decomposition, where the calculation time was defined as the computational time required for each process except the communication time. This table shows that the calcu395 lation time for processes differed greatly with radial dimension decomposition as the number of processes increased. This phenomenon occurred with all three implementations, including MPI. 


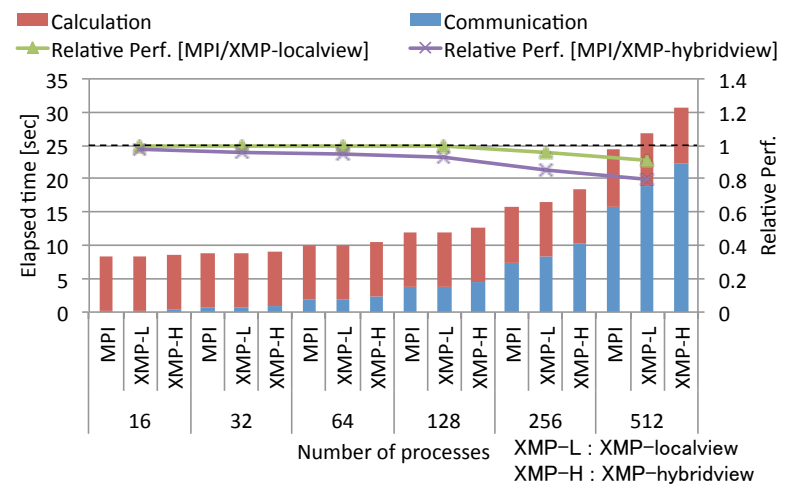

Figure 17: Elapsed time of the decomposition on radial dimension from 16 to 512 processes in weak scaling.

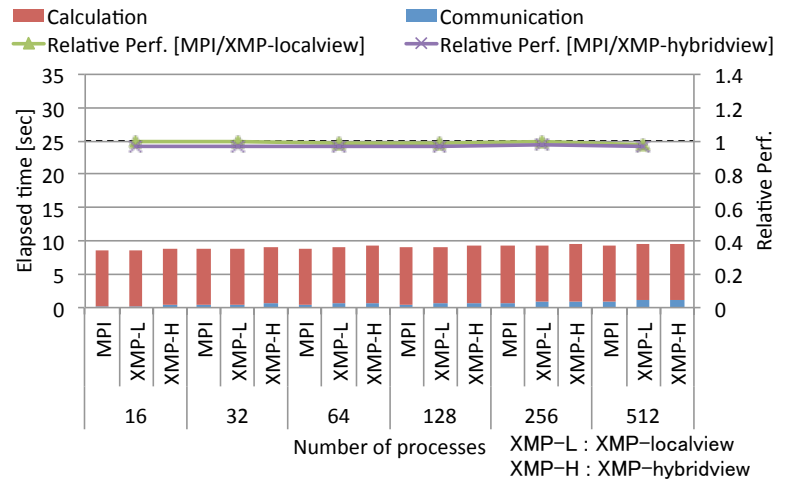

Figure 18: Elapsed time of the decomposition on particle dimension from 16 to 512 processes in weak scaling.

This may be explained by the method used to decompose the domain in the radial dimension. For other dimensions, it is easy to decompose the domain completely and equally for all processes. However, decomposition is complicated in the radial dimension because the domain volume varies in the inner part and outer part due to the torus form of the problem space. The volume and the corresponding grid size are calculated based on the formula used to describe the torus shape, which implies that an error is incurred during integer rounding to determine the number of grids. Table 5 shows that the number of total grid points assigned to the processes with the maximum and minimum calculation times differed greatly. Also, Figure 19 shows the breakdown of the minimum and maximum calculation times on the radial domain decomposition 512 processes. The difference between the calculation times of the grid-related 
Table 5: Load imbalance: maximum and minimum times required to calculate the processes with toroidal, radial, and particle decomposition [sec] (number of local grid points in each poloidal plane).

\begin{tabular}{l|c|c}
\hline \hline & \multicolumn{2}{|c}{ Toroidal } \\
\hline Processes & Minimum & Maximum \\
\hline 16 & $8.408406(19805)$ & $8.548204(19916)$ \\
32 & $8.440145(19805)$ & $8.541321(19916)$ \\
64 & $8.44846(19805)$ & $8.631631(19916)$ \\
128 & $8.511492(19805)$ & $8.718713(19916)$ \\
256 & $8.6418(19805)$ & $8.853517(19916)$ \\
512 & $8.865397(19805)$ & $9.109388(19916)$ \\
\hline \hline & \multicolumn{2}{|c}{ Radial } \\
\hline Processes & Minimum & Maximum \\
\hline 16 & $8.114932(10967)$ & $8.270015(16164)$ \\
32 & $8.083982(12104)$ & $8.539186(24200)$ \\
64 & $8.075058(14130)$ & $9.487029(33462)$ \\
128 & $8.070919(17422)$ & $11.014277(74745)$ \\
256 & $8.232447(23198)$ & $12.686402(141700)$ \\
512 & $8.763279(34522)$ & $16.508915(270844)$ \\
\hline \hline & \multicolumn{2}{|c}{ Particle } \\
\hline Processes & Minimum & Maximum \\
\hline 16 & $8.408406(19805)$ & $8.548204(19916)$ \\
32 & $8.406107(19805)$ & $8.558563(19916)$ \\
64 & $8.394203(19805)$ & $8.565195(19916)$ \\
128 & $8.394159(19805)$ & $8.562974(19916)$ \\
256 & $8.393343(19805)$ & $8.591214(19916)$ \\
512 & $8.390172(19805)$ & $8.641762(19916)$ \\
\hline
\end{tabular}

works, such as charge, push, poisson, field, and smooth as shown the detail in Section 4.2, increased on radial domain decomposition. During each time step, the computation of all processes must be bounded as a barrier operation and the increase in the integer rounding error according to the problem size (i.e., weak scaling) causes a greater load imbalance, which degrades the overall performance.

On the other hand, the communication time of XMP-localview and XMPhybridview on the radial dimension increases as the number of nodes increased compared with the MPI, as shown in Figure 17. We explored the number of send calls and each communication size because the performance of communication on XMP and MPI are reversed at about 65536 Bytes according to Figure 15. Figure 20 shows the number of send calls in process number 0 on each domain decomposition classified as the communication size of more than 65536 Bytes and 65536 Bytes or less. In the radial domain decomposition, the number of send calls at more than 65536 Bytes increases compared with the toroidal and particle decomposition. Therefore, the performance of XMP-localview and XMP-hybridview is degraded compared with MPI. The results were the XMPlocalview implementation obtains approximately the same performance as the MPI implementation while the performance degradation using XMP-hybridview is increased by up to $20 \%$ compared with the MPI implementation. Currently, we are under investigation why the number of send calls increased on the radial domain decomposition in weak scaling.

With strong scaling, Figures 21 and 22 show the elapsed time for both calcu- 


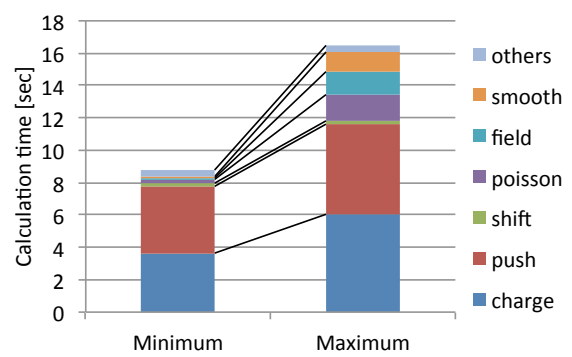

Figure 19: Breakdown of the minimum and maximum calculation times on the radial domain decomposition using 512 processes.

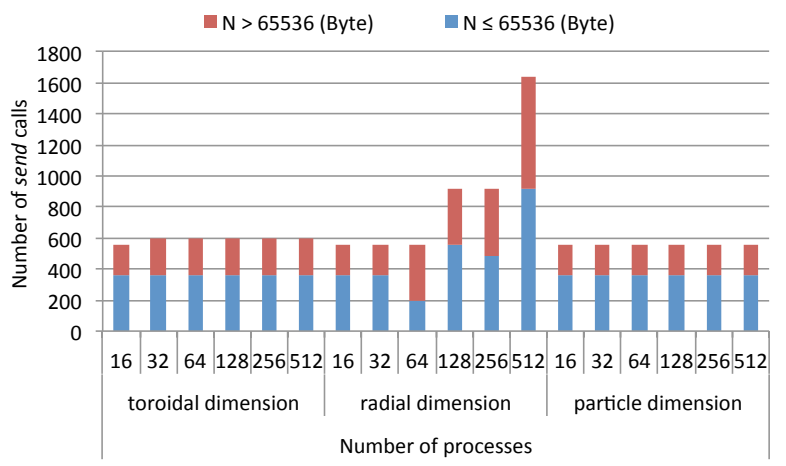

Figure 20: Number of send calls in process number 0 on each domain decomposition from 16 to 512 processes in weak scaling.

lation and communication of MPI, XMP-localview and XMP-hybridview, where the decomposition on the radial and particle dimensions, respectively. The performances of XMP-localview, and XMP-hybridview on the particle dimension are almost same compared with MPI, as shown Figure 22, while the elapsed time of the decomposition on the radial dimension increases as the number of nodes increased, as shown Figure 21.

We explored the number of send calls and each communication size same as 440 weak scaling. Figure 23 shows the number of send calls in process number 0 on radial and particle domain decomposition classified as the communication size of more than 65536 Bytes and 65536 Bytes or less. The number of send calls on the radial domain decomposition at 65536 Bytes or less increases compared with the particle decomposition. Therefore, the performance of XMP-localview and XMP-hybridview are increased compared with MPI on radial domain decomposition from 128 to 512 processes in strong scaling.

Figure 24 shows the elapsed time of the decomposition on radial and particle dimension, i.e., $2 \times 4 \times 2$ and $2 \times 2 \times 4$, ranged from 1 to 16 threads per 


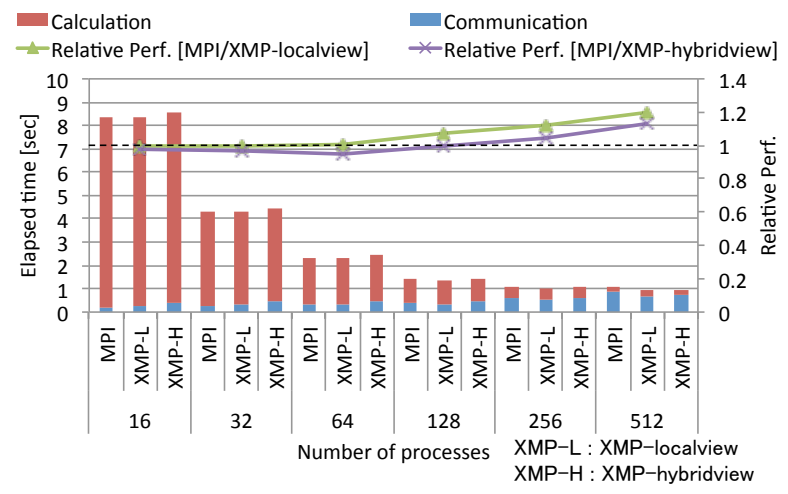

Figure 21: Elapsed time of the decomposition on radial dimension from 16 to 512 processes in strong scaling.

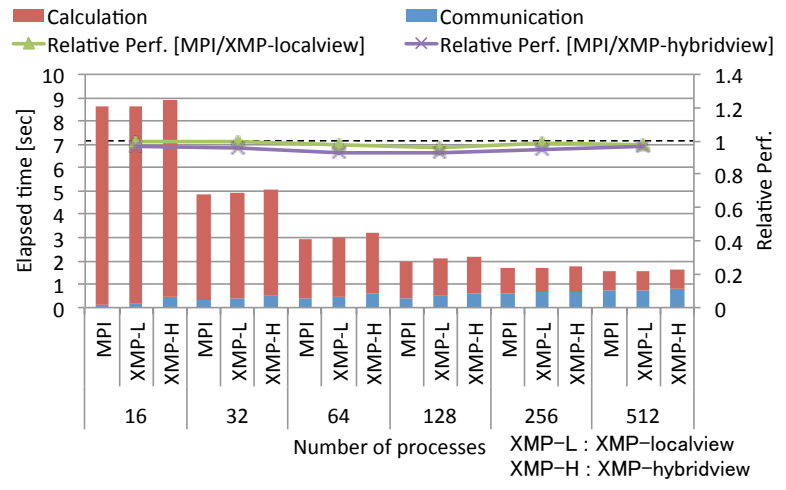

Figure 22: Elapsed time of the decomposition on particle dimension from 16 to 512 processes in strong scaling.

process using 16 nodes where one process ran on each node. The results were the performance of XMP implementation with thread parallelization is scaled the same as MPI.

\subsection{Productivity and Performance}

A good programming environment should facilitate high performance and high productivity, but high performance is sometimes obtained by low-level programming such as MPI, which unfortunately yields low productivity.

The XMP-localview implementation is simple and intuitive compared with MPI because the coarray communication is expressed in the form of an array assignment statement, as shown Figures 10 and 12. In coarray notation, the communication size and data are intuitively represented by array section and 


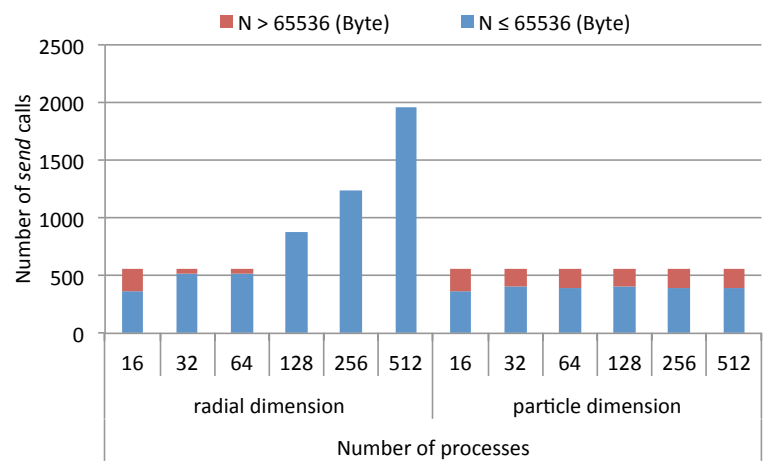

Figure 23: Number of send calls in process number 0 on each domain decomposition from 16 to 512 processes in strong scaling.

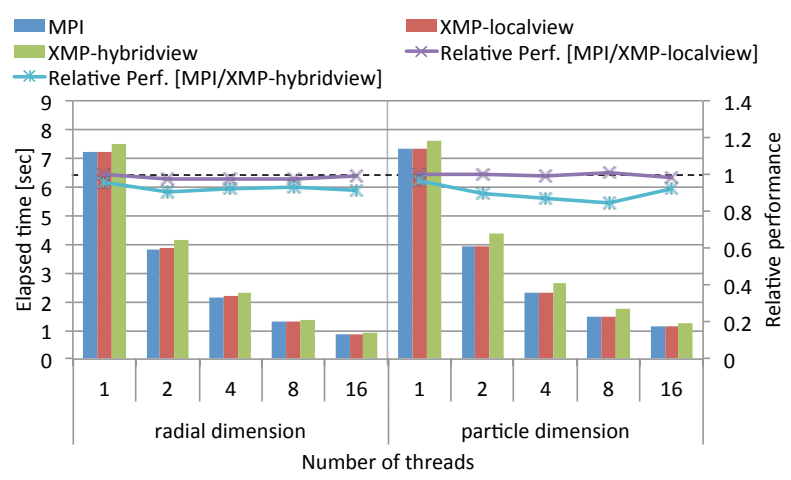

Figure 24: Elapsed time of the decomposition on radial and particle dimension from 1 to 16 threads.

460 comparable to that of the MPI version.

In XMP-hybriview, the global data structure required for the field data is described in the global-view model, which is almost the same as that in the serial code without particle calculation to communicate to the other process, and its data distribution is annotated by the directives, as shown in Figure 14. This improves the readability of the code because it is unnecessary for users to describe the many arguments on a line such as MPI APIs, thereby facilitating the easy maintenance of the program and simple parallelization from the original sequential code. For the global data structure, the communication with the 470 overlapped sleeve area in the distributed calculation domain can be described in only one line of the reflect directive, as shown in Figure 13.

Table 6 shows the delta Source Lines Of Code (SLOC) [24] for several implementations. This metric indicates how many lines of code changed from 
Table 6: Differences in the delta SLOC from Serial implementation for several different implementations of GTC-P.

\begin{tabular}{c|c|c|c|c}
\hline \hline & \multirow{2}{*}{ Serial } & \multirow{2}{*}{ MPI } & \multicolumn{2}{|c}{ XMP } \\
\cline { 4 - 5 } & & & localview & hybridview \\
\hline SLOC & 4110 & 5427 & 5398 & 5179 \\
\hline modified & - & 170 & 168 & 158 \\
added & - & 1319 & 1303 & 1112 \\
deleted & - & 2 & 15 & 43 \\
\hline Total delta SLOC & - & 1491 & 1486 & 1313 \\
\hline
\end{tabular}
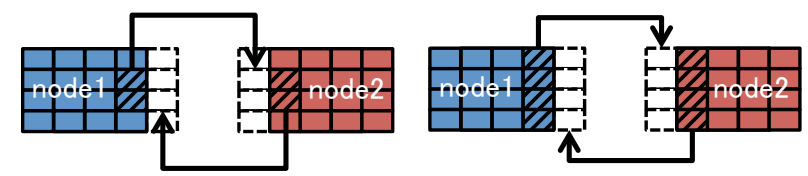

Figure 25: Updating of the sleeve area with MPI and XMP-localview (left), and XMPhybridview (right) in GTC-P. The hatching areas are communicated to nearby node.

the serial implementation of GTC-P, which shows modified, added, and deleted lines. Due to the reasons described above, the amount of code added and modified from the serial implementation is smaller with the XMP-hybridview implementation than the MPI implementation. In both of XMP implementations, the deleted lines are larger than MPI implementation because the explicit memory free is unnecessary for the distributed array and communication buffer for coarray in the global scope. In summary, XMP-hybridview implementation increases productivity.

The difference in performance between XMP-hybridview and MPI is attributable to the increase in the communication size of the reflect directive. The reflect directive is responsible for the communication designated as the sleeve area by the width clause, but it cannot update partially sleeve area. Figure 25 shows that the two-dimensional array is distributed to two nodes and exchanged the sleeve area in MPI and XMP implementations. In GTC-P, there is the communication pattern updated only inner sleeve area which is represented as the hatching areas in Figure 25 (left).

\section{Conclusion}

In this study, we implemented two versions of GTC-P, a large-scale nuclear fusion simulation code, using the global-view and local-view programming models in XMP for parallel programming languages, and we evaluated their performance and productivity. The first version, XMP-localview, only uses coarray communication in the local-view programming model, which simply replaces MPI point-to-point communication, except for collective communication such as MPI_Allreduce. The second version, XMP-hybridview, uses the distribution of the calculation domain and the reflect directive in the global-view 
programming model, as well as coarray communication for particle motion in the local-view programming model. Experimental evaluations showed that the XMP-localview implementation obtained approximately the same performance as MPI, whereas the XMP-hybridview implementation degraded the performance by $20 \%$. In addition, we obtained high productivity with the XMP implementation. In XMP-localview, the coarray notation is simpler and more

505 intuitive compared with MPI programming, and the XMP-hybridview allows more natural data expression for both static grid space data (in the global-view model) and dynamic particle data (in the local-view model), thereby increasing the readability of the code.

In the future, we will develop and perform the following investigations. First, we will develop the XMP hybrid-view implementation of GTC-P using the data distribution fixed at run-time. Second, we will evaluate the performance of the XMP implementation on a problem size that is compatible with ITER. Second, we will port our method to a GPU cluster using XcalablaACC[25] which is a hybrid model of the XcalableMP and OpenACC.

\section{Acknowledgments}

This research was partly supported by the JST-CREST program entitled "Research and Development of Unified Environment on Accelerated Computing and Interconnection for Post-Petascale Era." The authors are grateful for the utilization of the HA-PACS system at the Center for Computational Sciences, University of Tsukuba, as part of the Interdisciplinary Collaborative Research Program.

\section{References}

[1] UPC Consortium, Upc language specifications v1.3, https://upc-lang.org/ assets/Uploads/spec/upc-lang-spec-1.3.pdf, 2013 (accessed 16.03.09).

[2] R. W. Numrich, J. Reid, Co-array fortran for parallel programming, SIGPLAN Fortran Forum, Vol. 17, No. 2, New York, (1998) 1-31. doi:10.1145/289918.289920.

[3] P. Charles, C. Grothoff, V. Saraswat, C. Donawa, A. Kielstra, K. Ebcioglu, C. von Praun, V. Sarkar, X10: An object-oriented approach to non-uniform cluster computing, Proceedings of the 20th Annual ACM SIGPLAN Conference on Object-oriented Programming, Systems, Languages, and Applications (OOPSLA), New York, (2005) 519-538. doi:10.1145/1094811.1094852.

[4] XcalableMP Specification Working Group, Xcalablemp language specification version 1.2.1, http://www.xcalablemp.org/download/spec/xmp-spec1.2.1.pdf, 2014 (accessed 16.03.09). 
[5] J. Lee, M. Sato, Implementation and performance evaluation of xcalablemp: A parallel programming language for distributed memory systems, Proceedings of the 39th International Conference on Parallel Processing Workshops (ICPPW), San Diego, (2010) 413-420. doi:10.1109/ICPPW.2010.62.

[6] M. Nakao, J. Lee, T. Boku, M. Sato, Productivity and performance of global-view programming with xcalablemp pgas language, Proceedings of the 12th IEEE/ACM International Symposium on Cluster, Cloud and Grid Computing (CCGrid), Ottawa, (2012) 402-409. doi:10.1109/CCGrid.2012.118.

[7] S. Ethier, M. Adams, J. Carter, L. Oliker, Petascale parallelization of the gyrokinetic toroidal code, Proceedings of the 9th International Meeting on High Performance Computing for Computational Science (VECPAR), California, (2010) 1-9. doi:10.1.1.185.4712.

[8] Y. Shimomura, R. Aymar, V. Chuyanov, M. Huguet, R. Parker, Iter overview, Nuclear Fusion, Vol. 39, No. 9Y, Vienna, (1999) 1295-1308.

[9] DoE SCiDAC, UC Irvine, etc., Gyrokinetic toroidal code, http:// phoenix.ps.uci.edu/GTC/ (accessed 16.03.09).

[10] K. Tsugane, H. Nuga, T. Boku, H. Murai, M. Sato, W. Tang, B. Wang, Hybrid-view programming of nuclear fusion simulation code in the pgas parallel programming language xcalablemp, Proceedings of the 20th IEEE International Conference on Parallel and Distributed Systems (ICPADS), Hsinchu, (2014) 640-647. doi:10.1109/PADSW. 2014.7097864.

[11] Cray Inc, Chapel language specification version 0.98, http:// chapel.cray.com/spec/spec-0.98.pdf, 2015 (accessed 16.03.09).

[12] X. Liao, L. Xiao, C. Yang, Y. Lu, Milkyway-2 supercomputer: system and application, Frontiers of Computer Science, Vol. 8, No. 3, (2014) 345-356. doi:10.1007/s11704-014-3501-3.

[13] K. Madduri, K. Z. Ibrahim, S. Williams, E.-J. Im, S. Ethier, J. Shalf, L. Oliker, Gyrokinetic toroidal simulations on leading multi- and manycore hpc systems, Proceedings of 2011 International Conference for High Performance Computing, Networking, Storage and Analysis (SC), Seattle, (2011) 23:1-23:12. doi:10.1145/2063384.2063415.

[14] H. Sakagami, T. Mizuno, Compatibility comparison and performance evaluation for japanese hpf compilers using scientific applications, Concurrency and Computation: Practice and Experience, Vol. 27, (2002) 555-573. doi:10.1002/cpe.644.

[15] C. H. Koelbel, M. E. Zosel, The high performance fortran handbook, Vol. Cambridge, MIT Press, (1993). 
[16] R. Preissl, N. Wichmann, B. Long, J. Shalf, S. Ethier, A. Koniges, Multithreaded global address space communication techniques for gyrokinetic fusion applications on ultra-scale platforms, Proceedings of 2011 International Conference for High Performance Computing, Networking, Storage and Analysis (SC), Seattle, (2011) 78:1-78:11. doi:10.1145/2063384.2071033.

[17] RIKEN AICS, University of Tsukuba, Omni compiler project, http://omnicompiler.org/ (accessed 16.03.09).

[18] H. Murai, M. Sato, An efficient implementation of stencil communication for the xcalablemp pgas parallel programming language, Proceedings of the 7th International Conference on PGAS Programming Models, Edinburgh, (2013) 1-15.

[19] H. Nuga, A. Fukuyama, Kinetic modeling of the heating processes in tokamak plasmas, PhD Thesis, Kyoto Univ, (2011) 1-111.

[20] S. Ethier, W. M. Tang, Z. Lin, Gyrokinetic particle-in-cell simulations of plasma microturbulence on advanced computing platforms, Journal of Physics: Conference Series, Vol. 16, No. 1, (2005) 1-15.

[21] B. Wang, S. Ethier, W. Tang, T. Williams, K. Z. Ibrahim, K. Madduri, S. Williams, L. Oliker, Kinetic turbulence simulations at extreme scale on leadership-class systems, Proceedings of the International Conference on High Performance Computing, Networking, Storage and Analysis, Denver, (2013) 82:1-82:12. doi:10.1145/2503210.2503258.

[22] Center for Computational Sciences, University of Tsukuba, Ha-pacs project, http://www.ccs.tsukuba.ac.jp/eng/research-activities/projects/ ha-pacs/ (accessed 16.03.09).

[23] LBNL FTG, U.C. Berkeley, Gasnet specification version 1.8, http:// gasnet.lbl.gov/dist/docs/gasnet.pdf, 2006 (accessed 16.03.09).

[24] A. Stone, J. Dennis, M. Strout, Evaluating coarray fortran with the cgpop miniapp, Proceedings of 5th International Conference on PGAS Programming Models, Texas, (2011) 1-10.

[25] M. Nakao, H. Murai, T. Shimosaka, A. Tabuchi, T. Hanawa, Y. Kodama, T. Boku, M. Sato, Xcalableacc: Extension of xcalablemp pgas language using openacc for accelerator clusters, Proceedings of the First Workshop on Accelerator Programming Using Directives (WACCPD), New Orleans, (2014) 27-36. doi:10.1109/WACCPD.2014.6. 